\title{
Metastatic Prostate Cancer with Highest Reported PSA Level
}

\author{
Martha Orsolya, Tilinca Mariana*, Tataru Octavian Sabin, Chiujdea Sever, Balan Daniel, Vartolomei Mihai \\ Dorin
}

University of Medicine and Pharmacy Tirgu Mures, Romania

\begin{abstract}
Introduction: Prostate cancer is the sixth most common cancer in the world, the second most common cancer among men, and the most common cancer in men in Europe. Metastatic prostate cancer among young patients represents the rarest of the newly diagnosed prostate cancer, with few reports of cases with a longer survival. Case presentation: We present the case of a 59 year-old male who was referred with back pain over the last month. Digital rectal examination highlighted an enlarged and totally indurated prostate of $4 \times 4.5 \mathrm{~cm}$, while abdominopelvic X-rays showed osteoblastic metastases in the spine and pelvis bones. Laboratory examinations revealed a Prostate Specific Antigen level of $7941 \mathrm{ng} / \mathrm{ml}$. Prostate biopsy histology showed a bilateral prostate cancer with a Gleason score of 8 . Androgen deprivation therapy and daily administration of biphosphonates were prescribed. After two years of treatment, the Prostate Specific Antigen level decreases to $8 \mathrm{ng} / \mathrm{ml}$. Conclusions: We reported the highest Prostate Specific Antigen level in a patient under 60 years old with metastatic prostate cancer. Prostate cancer remains an important public health problem due to the aggressiveness of the disease and advanced stage upon diagnosis. Prostate Specific Antigen is mandatory to evaluate, to have a reference level in order to prevent metastatic prostate cancer in young patients at diagnosis.
\end{abstract}

Keywords: highest PSA, prostate cancer, metastasis, young

Received: 30 August 2016 / Accepted: 14 September 2016

\section{Introduction}

Prostate cancer $(\mathrm{PCa})$ is the sixth most common cancer in the world, the second most common cancer among men, and the most common cancer in men in Europe, North America, and parts of Africa [1]. In 2000, there were 513,000 newly diagnosed cases worldwide, while in 2012 there were 1.1 million. This suggests an increased incidence of prostate cancer in the past decade [2].

Prostate-specific antigen (PSA) is a protein produced by the epithelial cells of the prostate gland. Its main attribution is to liquefy the semen in the seminal coagulum allowing the sperm to move freely. PSA is present in the serum in small quantities in healthy people, but it has an elevated level in case of prostate cancer or other prostatic disorders.

Even if $\mathrm{PCa}$ is an important health problem worldwide, screening based on Prostate-Specific Antigen (PSA) determination seems to increase the overall health care costs and early detection of prostate cancer, leading to an earlier treatment with several years before regular occurrence. Decision analysis models have been used to evaluate the effectiveness of prostate cancer screening and treatment, but little or no benefit was found [3].

Metastatic Pca (mPCa) among young patients represents few of the newly diagnosed PCa, most of the clinical trials showed a mean survival after diagnosis up to 3 years, with few case reports of longer survival [4]. Very high PSA levels

${ }^{*}$ Correspondence to: Mariana Tilinca

E-mail: mariana.tilinca@umftgm.ro in $\mathrm{mPCa}$ at diagnosis are rarely reported and none referring to a Caucasian male under 60 years old $[5,6]$.

\section{Case presentation}

We present the case of a 59 year-old Caucasian male patient who was referred to the Urology Emergency Unit of the Mures County Hospital suffering from back pain over the last month. Abdominal ultrasound examination revealed no pathological findings, but digital rectal examination highlighted an enlarged and totally indurated prostate of $4 \times 4.5 \mathrm{~cm}$, while abdomino-pelvic X-rays showed osteoblastic metastases in the spine and pelvis bones. The patient was sent to the laboratory to undergo some investigations, including a PSA-level assessment. Laboratory results revealed a PSA level of $7941 \mathrm{ng} / \mathrm{ml}$. This represented the mean level of two PSA determinations. After the patient signed an informed consent, he was scheduled for an ultrasound guided prostate biopsy at the Urology Clinic. A 12 core prostate biopsy was performed using a Vienna nomogram. Biopsy fragments were sent for histological examination, standard histology methods and hematoxylin eosin stain were used. The examination of the tissue fragments established a diagnosis of bilateral Prostate Adenocarcinoma with a Gleason score of 8 . In the right lobe Gleason score was $8(4+4)$ and in the left lobe the Gleason score was $7(4+3)$ in all biopsies with a bilateral perineural invasion. Scintigraphy was not performed due to a long time recovery after a cervical fracture. Follow-up protocol was done according to EAU guidelines for clinical T3 PCa 
with bone metastases. Surgical removal of the testes was proposed as one of the therapeutic options but the patient chose medical castration. Androgen deprivation therapy (ADT) was started at the Oncology Department of our hospital. The oncology treatment included anti-androgen therapy (Bicalutamid) $50 \mathrm{mg}$ every day, Leuprolein (Eligard) at an interval of 6 months and daily administration of bi-phosphonates for preventing the loss of bone mass.

One year follow-up computed tomography (CT) examination showed: minimal bilateral gynecomastia; a slightly increased thyroid gland; no sign of pulmonary thromboembolism; minimal pulmonary fibrosis; the lack of pulmonary nodules; trachea and bronchi with clear passage; normal mediastinum; no sign of pericardial fluids; 20 $\mathrm{mm}$ large duodenal diverticulum in D2 portion; normal conformed liver and gall bladder. The pancreas, suprarenal glands, spline, kidneys were without any CT-observed modifications. The bladder and bladder-wall were also in normal condition; the dimensions of the prostate were $31 / 23 \mathrm{~mm}$ with a regular outline; the seminal vesicles were symmetrically relaxed; the level of the hydrocele was normal. No abdominal-pelvic pathologic lymph-nodes as well as no atheroma in the aorta were detected. Sclerotic bone metastasis in the skeletal system: scapular-belt, thoracic box, spine, pelvis, femoral head - no pathological bone fractures and no stenosis in the spinal canal (Figure 1). After one year of treatment, the PSA level decreased to 8ng/ $\mathrm{ml}$ and the patient's clinical status suggested the benefit of the treatment.

\section{Discussion}

In 2013, the American Urological Association (AUA) changed the guidelines concerning prostate cancer screening based on the high rate of insignificant $\mathrm{PCa}$ detection among men younger than 55 years, recommending PSA testing only in case of significant risk factors: family history of prostate cancer or African ethnicity [7]. There are two trials in literature - The Prostate, Lung, Colorectal and Ovarian (PLCO) screening trial and the European Randomized Study of Screening for Prostate Cancer (ERSPC) one which represent the highest level of evidence regarding PSA screening $[8,9]$. In the PLCO trial, after 13 years of follow-up, there was no evidence of improved mortality

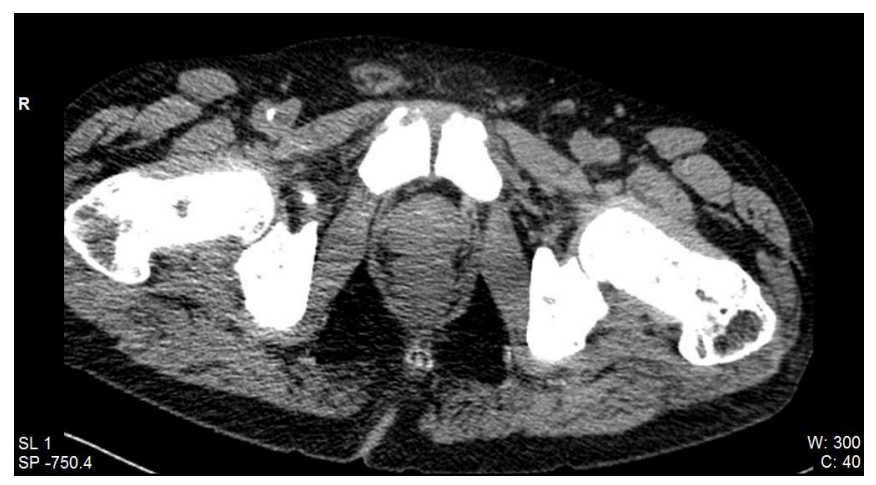

Fig. 1. Prostate CT after one year hormonal treatment rates in case of organized annual screening compared with opportunistic screening. On the other hand, the ERSPC trial showed a benefit regarding a reduction in metastatic disease rates at diagnosis in the screening arm, and a mortality reduction by almost 3 years $[10,11]$.

Despite advances in prostate cancer diagnosis and management, morbidity from prostate cancer is still high, approximately $20 \%$ of men present with advanced or metastatic disease, while 29,000 men continue to die of prostate cancer each year.

Available screening tests (for example prostate specific antigen) can detect early stage disease but there is no evidence that clinical outcomes are improved by early cancer detection. The potential harms of performing screening for 28 million men older than 50 years include unnecessary interventions for thousands of men without any disease or with cancer but no clinical manifestations [12].

Androgen-deprivation therapy (ADT) through surgical gonadectomy is equally effective as medical castration in controlling prostate cancer (PCa). However, Gonadotropin-releasing hormone agonist therapy was associated with higher risks of cardiac-related complications compared with orchiectomy, but usually as in our case clinical decision making for medical castration therapy is also based on the patient's choice $[13,14]$.

Since the benefit of prostate-specific antigen (PSA) screening remains controversial, new non-invasive biomarkers for prostate carcinoma ( $\mathrm{PCa}$ ) are still required, new evidence showed that microRNAs (miRNAs) in whole peripheral blood can separate patients with localized prostate cancer from healthy individuals [15].

\section{Conclusion}

We reported the highest PSA level in a patient under 60 years old with mPCa. Prostate cancer remains an important public health problem due to the aggressiveness of the disease and advanced stage of the disease upon diagnosis. Even if screening raises the costs of the disease, a PSA level in men over 50 years of age, it is mandatory to have a reference level in order to prevent $\mathrm{mPca}$ in young patients at diagnosis.

\section{Acknowledgment}

This paper was supported by a research Grant won at University of Medicine and Pharmacy, Târgu Mureș, Romania, contract No. 17800/9/22.12.2015.

\section{Conflict of interest}

Nothing to declare.

\section{References}

1. Grönberg H. Prostate cancer epidemiology. Lancet Lond Engl. 2003;361(9360):859-864.

2. Globocan 2012. Available from: http://globocan.iarc.fr/Default.aspx

3. Benoit RM, Naslund MJ. The socioeconomic implications of prostatespecific antigen screening. Urol Clin North Am. 1997;24(2):451-458.

4. Krpina K, Markić D, Rahelić D, Ahel J, Rubinić N, Španjol J. 10-year 
survival of a patient with metastatic prostate cancer: Case report and literature review. Arch Ital Urol Androl. 2015;87(3):252-3. doi: 10.4081/ aiua.2015.3.252.

5. Slipman CW, Patel RK, Siegelman ES, et al. Metastatic prostate cancer to the spine and a PSA of 5666: a case report. Pain Physician. 2001;4(4):317-321.

6. Persec Z, Persec J, Sović T, Romic Z, Bosnar Herak M, Hrgovic Z. Metastatic prostate cancer in an asymptomatic patient with an initial prostate-specific antigen (PSA) serum concentration of $21,380 \mathrm{ng} / \mathrm{ml}$. Onkologie. 2010;33(3):110-112. doi: 10.1159/000277743.

7. Dantanarayana ND, Hossack T, Cozzi P, et al. Men under the age of 55 years with screen detected prostate cancer do not have less significant disease compared to older men in a population of patients in Australia. BMC Urol. 2015;15(1):124. doi: 10.1186/s12894-015-0117-3.

8. Andriole GL, Crawford ED, Grubb RL, et al. Prostate cancer screening in the randomized Prostate, Lung, Colorectal, and Ovarian Cancer Screening Trial: mortality results after 13 years of follow-up. J Natl Cancer Inst. 2012;104(2):125-132. doi: 10.1093/jnci/djr500.

9. Schröder FH, Hugosson J, Roobol MJ, et al. Screening and prostatecancer mortality in a randomized European study. $\mathrm{N}$ Engl $\mathrm{J}$ Med. 2009;360(13):1320-1328. doi: 10.1056/NEJMoa0810084

10. Buzzoni C, Auvinen A, Roobol MJ, et al. Metastatic Prostate Cancer
Incidence and Prostate-specific Antigen Testing: New Insights from the European Randomized Study of Screening for Prostate Cancer. Eur Urol. 2015;68(5):885-890. doi: 10.1016/j.eururo.2015.02.042.

11. Schröder FH, Hugosson J, Carlsson S, et al. Screening for prostate cancer decreases the risk of developing metastatic disease: findings from the European Randomized Study of Screening for Prostate Cancer (ERSPC). Eur Urol. 2012;62(5):745-752. doi: 10.1016/j.eururo.2012.05.068.

12. Woolf $\mathrm{SH}$. Public health perspective: the health policy implications of screening for prostate cancer. J Urol. 1994;152(5 Pt 2):1685-1688.

13. Sun M, Choueiri TK, Hamnvik O-PR, et al. Comparison of GonadotropinReleasing Hormone Agonists and Orchiectomy: Effects of AndrogenDeprivation Therapy. JAMA Oncol. 2016;2(4):500-507. doi: 10.1001/ jamaoncol.2015.4917.

14. Nyman CR, Andersen JT, Lodding P, Sandin T, Varenhorst E. The patient's choice of androgen-deprivation therapy in locally advanced prostate cancer: bicalutamide, a gonadotrophin-releasing hormone analogue or orchidectomy. BJU Int. 2005;96(7):1014-1018.

15. Leidinger $P$, Hart M, Backes $C$, et al. Differential blood-based diagnosis between benign prostatic hyperplasia and prostate cancer: miRNA as source for biomarkers independent of PSA level, Gleason score, or TNM status. Tumour Biol. 2016;37(8):10177-10185. doi: 10.1007/s13277016-4883-7. 